\title{
Research on the Running-up and Take-off Technique of Long Jump in Athletics Teaching
}

\author{
Zhang ZongHao \\ College of Physical Education \\ Soochow University, Su Zhou, China \\ 786122448@qq.com
}

\begin{abstract}
Along with the continuous development of sport science, people's understanding of long jumps is strengthened. Under such backgrounds, this paper elaborates relevant running-up and take-off technique in long jump, hoping to contribute to better implement athletics teaching.
\end{abstract}

Keywords- Athletics Teaching; Long Jump; Running-up Technique; Take-off Technique

Long jump, as a sport focusing on power, speed, and technique has always been one of the projects for people to push their limits. The world record for men's long jump is $8.95 \mathrm{~m}$ created by an American famous player called Mike Powell in Tokyo, 1991, while the world record for women is $7.52 \mathrm{~m}$ created by famous former Soviet Union player called GALINA GHISTYAKOVA in Leningrad (St. Petersburg) 1988. Because of different physical qualities, there is a big gap between Chinese long jumpers and world higher level players. How to keep steady rise in terms of long jump result is a problem concerned by relevant scholars remaining to be solved. This paper analyzes and discusses about relevant techniques and training methods of running-up and takeoff and meanwhile analyzes the long jump in athletics teaching for your reference.

\section{THE BASIC CONCEPT AND CHARACTERISTICS OF LONG JUMP}

Long jump is also known as running broad jump, which is an important field event. The technique movements of long jump are made up of run-up, skipping, soaring, and falling to the ground. In the course of long jump, single-foot take off is frequently adopted and then the player shall fall to the ground and the jump distance is used to determine the ranking. The basic characteristic of long jump is the combination of the most powerful jump and fastest running speed so as to achieve the maximum distance.

\section{RUNNING-UP TECHNIQUE IN LONG JUMP}

\section{A. Running-up Speed}

The fast running-up speed can help players obtain the maximum take-off speed. According to relevant statics, among all influential factors, the running-up speed occupies $70 \%$ and in Men's High Jump, the correlation coefficient between running-up speed and high jump result is 0.943 . Even though the absolute speed determines whether there will be maximum approach speed or not, it is not absolute because in reality, there is an example of absolute low speed igniting high speed running-up. The key of perfectly combining these two is whether the speed in the running-up can be transferred into power for taking-off. [1]

\section{B. Running-up Distance}

Concerning the length of running-up, a proper distance should be chosen so as to have the fastest speed which is a problem easily ignored. In the course of running-up, if the distance is not enough, the speed is low and if the distance is too long, unnecessary physical consumption is wasted which will affect the taking-off speed. In general training and teaching the running-up distance is between $35-40 \mathrm{~m}$ for men and $30-35 \mathrm{~m}$ for women. However, with the changes of long jump result, training level and absolute speed, the running-up distance can be properly changed and reasonable changes can help to have the maximum running-up speed. ${ }^{[2]}$

\section{2.3 Running-up Starting Method}

In terms of running-up starting, there are progressive and standing forms. According to relevant data analysis, the success rate of adopting standing running-up is around $80 \%$ and the success rate of adopting progressive running-up is below $60 \%$. In the course of standing running-up, the physical postures have strong control capacity and therefore, knee-joint distance, upper body angel of inclination front, knee-joint bending angle, and two feet standing shall be same. As a result, the ground force is guaranteed to which can improve the success rate of taking-off. Even though the progressive form does not have strong control capacity, it can easily ignite faster speed which is flexible and that's why most of excellent players choose such form.

\section{Step Length and Frequency in the Course of Running-up}

When take off, the body gesture should be strictly controlled. What's more, when the running-up speed is steadily improved, the speed and rhythm frequency is also increased. In order to achieve the coherence, players have to accelerate the step frequency in the spring stage, trying the utmost to obtain the maximum. However, the stride length should not be increased in acceleration process because if the stride frequency is accelerated and 
the stride length is decreased, the speed cannot be increased and will lead to mistakes caused by nervous emotion as well as rhythm imbalance. In addition, if the length is increased and frequency is slowed, the takingoff shall be affected and even the striding mark will be affected.

\section{E. Run-Up Rhythm}

Good running-up rhythm is perfect combination of stride length and frequency. Running-up rhythm directly affects the length and frequency, which also greatly affects the stability and accuracy of running-up. Running-up rhythm is a kind of inertial steady rhythm trained for a long time. If the running-up changes, the accuracy shall be decreased which will then affect the long jump result. Therefore, if players want to full play the speed advantage, they have to be trained and guided, which should be the premise and guarantee for successfully completing taking-off. Only when the players correctly complete the last six steps, can they accurately take off so as to have a good long jump result. ${ }^{[3]}$

\section{THE TRAINING METHOD FOR RUNNING-UP TECHNIQUE}

In order to achieve the maximum effect of runningup, scientific training as well as teaching activities is important, which is the only means to obtain the maximum effect. This chapter mainly puts forward several opinions in terms of running-up training approaches from the perspectives of running-up distance, rhythm, and accuracy.

\section{A. Choose Proper Running-up Distance}

The correct running-up distance should be in accordance with the speed of players so as to take full advantage of their potential. If the running-up distance is too long, then the players waste their physical strength in advance and in the last steps, they cannot have the fastest speed and as a result the whole long jump rhythm and result shall be affected. In contrast, if the running-up distance is too short, players also cannot have the fastest speed while taking off which will also bring about bad influence on the result. Then, which principle shall be obeyed? According to the current approach, the runningup distance and steps can be determined based on the average speed from $30 \mathrm{~m}$ to $100 \mathrm{~m}$.

\section{B. Cultivate Correct Running-up Rhythm}

1) Choose 40-60m running-up distance. In the course of training, we have to ask players to keep correct and steady speed so as to rhythmically and systematically improve the stride length, frequency and speed.

2) Choose proper downhill running and flat ground running. Based on different situations of players, 10-14 steps downhill running distance and 6-8 flat ground running distance can be chosen. What's more, players are asked to keep steady and accelerated speed based on the speed inertia of downhill. ${ }^{[4]}$
3) Choose 20-40m distance to run. In terms of this training, the fewest strides are needed with wider stride length in order to maintain quick speed and frequency.

4) The metronome is adopted to carry out systematic exercise, which asks players to be trained based on the external metronome.

\section{Improve the Accuracy of Running-up}

Enhance psychological training. Systematic psychological training can help players solve the following three problems. To begin with, establish confidence, cultivate bold spirit; secondly, help players establish stable psychological states; thirdly, the systematic training and exercising can comprehensively improve the running-up rhythm.

Combine running-up exercising and taking-off training. A solid belief has to be established and the aim of running-up is to obtain the longest long jump distance. With the deepening of running-up training, a combination of running-up and taking-off exercising should be strengthened so as to guarantee the accuracy and effectiveness and the separate training should be undesirable.

In the course of running-up exercising, the accuracy should be guaranteed because when the competitive state is constantly improved the possibility of breaking the rules is also improved. Therefore, in the course of training, the pedal distance should be correct so as to achieve the optimal effect.

\section{TAKING-OFF TECHNIQUE IN THE LONG JUMP}

Taking-off is the key sector to converts running-up speed into long jump distance. The jumping speed, running-up speed and angle all play a critical role in the long jump result. Whether the running-up speed can be converted into maximum taking-off speed or not depends on whether the jumping angle is scientific and reasonable or not, which is also greatly affected by the taking-off technique. Therefore, in the taking-off stage, the quick speed is the premise to create scientific takingoff.

\section{A. Take-off Preparation}

Taking-off is a critical sector for long jump, which is closely related to running-up. The final sprint stage of running-up should make full preparation for taking-off. In order to improve the speed of using rate or running-up, proper gravity descending should be done. 100 is taken as the standard and comparing to the gravity descending height in last three stages, only $7 \%$ gravity descending height is adopted in the last second step and in the last step $10 \%$ gravity descending height should be done. ${ }^{[5]}$

\section{B. Take-off Landing}

Positive landing can make preparation for taking off while decreasing ground reaction force. Positive, powerful, active landing can guarantee high level speed.

\section{Buffering}

Buffering refers to the stage of obtaining the maximum knees bending degree while landing off. As an important sector in terms of taking-off technique and the quality of buffering plays a crucial role in affecting 
the taking-off angle and quickly completing the buffering is the key to improve speed. What's more, buffering is to convert the dynamic energy in the running-up stage into the potential energy of bending and finally into the speed force.

\section{Stretching}

The stretching stage starts to maximize the knee bending degree and completes in jumping. In the course of stretching, the body moves ahead quickly and the stretching action directly affects the jumping speed and direction. Full preparation of stretching shall ignite quicker speed and larger jumping angle which can help to achieve good result.

\section{E. Swinging}

What's more, the swinging action can help to achieve good effect in terms of taking-off, jumping speed and ground force decreasing. Therefore, the coordination of swinging and stretching should be highlighted while take off. Systematic swinging exercising can comprehensively improve the long jump result. ${ }^{[6]}$

\section{TECHNICAL TRAINING METHODS FOR TAKING-OFF}

The take-off exercising mainly aims to generate explosive and expansion force of muscle based on specialized force and speed training. Only by systematic training, the running-up speed can be converted into the maximum distance and force requirement.

\section{A. Take-off jump support training}

Adopt squatting training methods. Based on the gravity position of players, relevant bench height can be determined and once the bottom touches the bench, players can push their body and stretch their knee, ankle, and hip.

Players can carry on barbell which can be affordable and then conduct squatting exercising with weight and guarantee $145^{\circ}$ keen bending angle with about $3-5$ s relative stillness and then stretch knee, ankle and hip.

After studying on relevant researches, we can find out that the muscle operation principle of static training method is different from take-off jump period. The main reason is that in the take-off jump period, the jumping is the follow-up action of buffering and in the course of training, there is direct explosive action which asks consistency between performance essence, scope as well as frequency and take-off jumping structure. Only by systematic and scientific training, the training effect and efficiency can be guaranteed.

\section{B. Training to Improve Take-off Jump Explosive Force}

a) Force explosive training. In general, standing multi-stage long-jump, standing long-jump, multi-stage frog jump, and standing three-step jump are adopted in force explosive training so as to ignite the explosive force of players. In the course of training, people are standing and slow sped can be adopted to complete the goal so as to achieve the optimal effect of training. ${ }^{[7]}$ b) Speed explosive training. In the course of speed explosive training, shot running-up hopping (4-6 steps quick running-up strides to complete 5-10 level hopping) as well as skipping column by single runningup (4-8 running up strides to fully complete the jumping and skipping the column based on relevant conditions.)

This training method can obviously stimulate and improve players' jumping yet with problems as squat that is to say there is gap between muscle circumstances and real jumping. Therefore, in training methods selection, the combination of jumping direction and ability should be strengthened so as to guarantee that the training is orderly carried out under scientific and reasonable circumstances.

c) Super-Equal-Length Training. Super-equallength training can be regarded as the best means to train players' speed and force. Depth jumping exercise mainly include jumping from high to down as well as jump from down to high and bounce jumping. The depth jumping exercise mainly aims to draw the muscle and realize the geometric growth of force. Because in jumping from high to down, the muscle is on the strain and the power is about 20 times of weight. Therefore, concerning such training methods, the step by step principle should be insisted without the idea of adventurist.

The essentials of performance as well as principles of squatting exercising are similar with which can achieve the maximum speed based on the conversion of buffering and stretching. In the course of training, the distance selection should be focused on so as to increase the distance with the deepening of exercising and meanwhile the landing technique as well as angle should be trained. Such training methods can strengthen the force of taking off which shall also greatly affect the overall result. Therefore, proper selection will ignite positive effect on the taking off effect.

\section{CONCLUSION}

In long jump, the success of running-up and taking off directly affect the result. Therefore, in athletics teaching we have to pay attention to how to improve take-off and running-up techniques. The reasonable and scientific long jump technique is the key to have excellent long jump result. So, the running-up as well as taking-off rhythm should be scientific, which is also the critical point to achieve success. This author expects that sky rocketing professional scholars can research on this project and put forward suggestions concerning shortages in this paper, hoping to contribute to Chinese athletics education.

\section{REFERENCES}

[1] Qi ZHANG,Hao YANG,Yuguang WEI et al.Selection of Destination Ports of Inland-Port-Transferring RHCTS Based on Sea-Rail Combined Container Transportation[C].//nnovation and sustainability of modern railway: Third international symposium on Innovation and sustainability of modern railway (ISMR 2012), September 20-21, 2012, Nanchang, China.2012:675-680.

[2] Zang Jibin. Review of long-jump techniques in physical education [J]. China Education Innovation Herald, 2010, (3).

[3] Zhang Chuanping, Liu Jiguan. Discuss on the long-jump techniques and training methods $[\mathrm{J}]$. SME management and technology, 2011, (24). 
[4] Hangying Lou,Guozheng Wang.The 2 High Horizontal Man Sportsman Run-up Final Step Sport Credits of Long Jump of Horizontal Speed Loss Reason[C].//Nanotechnology and Computer Engineering.2010:728-732.

[5] Liu Lei. Brief analysis on the deliberated index selection for long jump techniques [J].Electronic education for New Era Education, 2015, (4):93.
[6] Zou, Kening. Mathematical analysis of function and mechanism of run-up and take-off in the long jump[C].//2011 IEEE 2nd International Conference on Computing, Control and Industrial Engineering. v.2.2011:151-154.

[7] He Xiangrong. Discuss on the effective factors which can improve long jump techniques [J]. Contemporary Sports Technology, $2014,4(2)$ 